\title{
Characteristics of Recent Articles Published in the Korean Journal of Radiology Based on the Citation Frequency
}

\author{
Yeon Hyeon Choe, MD, PhD ${ }^{1,2}$ \\ ${ }^{1}$ Department of Radiology, Samsung Medical Center, Sungkyunkwan University School of Medicine, Seoul, Korea; ${ }^{2}$ HVSI Imaging Center, Heart \\ Vascular Stroke Institute, Samsung Medical Center, Sungkyunkwan University School of Medicine, Seoul, Korea
}

Citation frequency (CF) is considered the measure of academic interest and scientific value of an article, and it may reflect the current and future practice of radiology and medicine. The contents of highly-cited articles may represent recent academic trends in medicine and science in an academic community related to the journal. Articles with high CF may provide researchers an insight on areas of research interest.

According to the annual CFs of 398 articles published in 2017-2019, reported in the Journal Citation Reports (Web of Science core collection) by Clarivate Analytics as of October 31, 2020, there were $12(3.0 \%)$ very highly-cited articles (annual CF, 10-51), 15 (3.8\%) highly-cited articles (annual CF, 5.0-9.75), 37 (9.3\%) intermediately-cited articles (annual CF, 3.0-4.67), and 276 (69.3\%) low-citation articles (annual CF, 0.25-2.67). Fifty-eight articles (14.6\%) were not cited at all.

Fifty-one articles (12.8\%) were cited 10 or more times in total. The average CF of original articles was 4.17 , while that of review articles was 14.01 . Thirty-five $(12.3 \%)$ of 284 original articles were not cited at all, while four (5.6\%) of the 72 review articles were not cited. The numbers of

Received: November 5, 2020 Revised: November 5, 2020

Accepted: November 5, 2020

Corresponding author: Yeon Hyeon Choe, MD, PhD, Department of Radiology, Samsung Medical Center, Sungkyunkwan University School of Medicine, 81 Irwon-ro, Gangnam-gu, Seoul 06351, Korea.

- E-mail: yhchoe@skku.edu

This is an Open Access article distributed under the terms of the Creative Commons Attribution Non-Commercial License (https://creativecommons.org/licenses/by-nc/4.0) which permits unrestricted non-commercial use, distribution, and reproduction in any medium, provided the original work is properly cited. articles with total $\mathrm{CF} \geq 10$ were $26(36.1 \%)$ for review articles and $25(8.8 \%)$ for original articles.

The top citation article (CF, 204) was related to deep learning. The number of original articles in areas of subspecialties or imaging technologies with total $\mathrm{CF} \geq 10$ were six articles in gastrointestinal/hepatobiliary imaging or intervention, three in thyroid imaging or intervention, and three in breast imaging. The number of review articles in areas of subspecialties or imaging technologies with total CF $\geq 10$ were eight articles in society guidelines or consensus documents, six in gastrointestinal/hepatobiliary imaging or intervention, and five in thyroid imaging or intervention.

\section{Conflicts of Interest}

The author has no potential conflicts of interest to disclose.

ORCID iD

Yeon Hyeon Choe

https://orcid.org/0000-0002-9983-048X 